\title{
An Extrinsic Component Parameter Extraction Method for High Power RF LDMOS Transistors
}

\author{
J. Wood, D. Lamey, M. Guyonnet, D. Chan, D. Bridges, N. Monsauret ${ }^{\dagger}$, and P. H. Aaen \\ Freescale Semiconductor, Inc, RF Division, Tempe, AZ and Toulouse, France ${ }^{\dagger}$
}

\begin{abstract}
A new extrinsic network and extrinsic parameter extraction methodology is developed for high power RF LDMOS transistor modeling. This new method uses accurate manifold deembedding using electromagnetic simulation, and optimization of the extrinsic network parameter values over a broad frequency range. The new extrinsic network accommodates feedback effects which are observed in high power transistors. This improved methodology allows us to achieve a good agreement between measured and modeled $S$-parameters in the frequency range of 0.5 to $6 \mathrm{GHz}$ for different bias conditions. Large-signal verification of this new model shows a very good match with measurements at $2.14 \mathrm{GHz}$.
\end{abstract}

\section{INTRODUCTION}

Accurate nonlinear transistor models are nowadays considered to be prerequisite to a successful $\mathrm{RF}$ and microwave circuit or product design. Even as the complexity of the wireless circuits and systems continues to increase, there is continued pressure to shorten the design cycle and improve time-tomarket, improve performance, and to meet the tight regulatory constraints on spurious emissions. Traditional empirical design practices are being replaced by computer-aided design approaches, which require accurate transistor models.

The transistor model typically consists of two parts: the intrinsic model, which comprises the nonlinear elements or functions describing the operation of the transistor, and the extrinsic components, which account for the metallizations, substrate, and semiconductor that connect the intrinsic model to the outside world. The intrinsic components or functions are generally voltage-dependent, describing the nonlinear behavior of the device. The extrinsic components are usually considered to be independent of voltage, that is, passive components.

The nonlinear (intrinsic) model has received considerable attention from the modeling community for the development of accurate functional descriptions of the transistor's properties. However, the careful identification of the components of the extrinsic network is also required for accurate transistor modeling, so that the extrinsic network can be de-embedded accurately to enable access to the intrinsic transistor.

Further, the extrinsic network plays a significant role in determining the scaling rules for the complete transistor model. The extrinsic network has to describe the electrical behavior of the device layout, and how this layout changes with device size determines how the extrinsic network component values scale. In $\mathrm{RF}$ power transistors, this is a significant consideration: the transistor can often have a total gate width of over 100 $\mathrm{mm}$ : this is impossible to model in a traditional small-signal S-parameter environment - the RF powers are too high, and

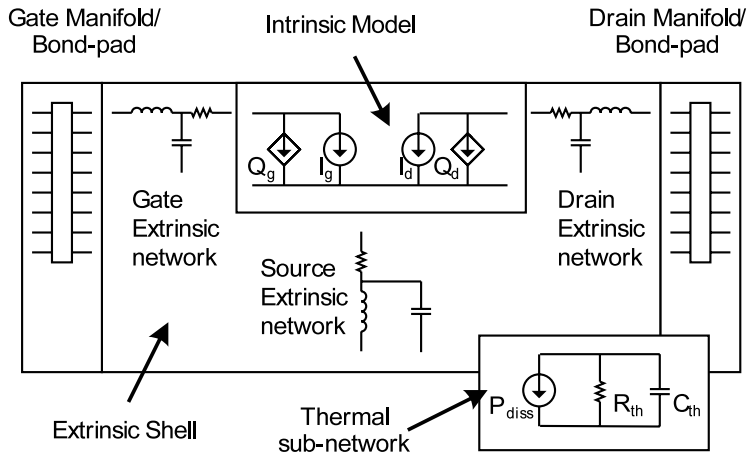

Fig. 1. Block representation of the transistor model architecture

the device impedances are too small to permit measurements of sufficient accuracy for model extraction, if indeed the device is stable in a $50 \Omega$ environment. It is necessary instead to model a smaller device structure, and scale the resulting model to the appropriate size. We have adopted a segmentation approach to deriving the transistor model. We choose a model structure that can be split or segmented in a way that can be defined by RF measurement reference planes, allowing each part or shell of the structure to be measured and modeled independently, and enabling the complete model to be constructed from these parts with confidence [1]. The block diagram of the model structure is shown in Fig. 1.

In this paper we shall describe a methodology for extraction of the passive parts of the model: the input and output feed or manifold structures, and the extrinsic passive network. The manifold structure, shown in Fig. 2, is modeled using electromagnetic simulations, and the model is validated against measured data from test structures. The manifold is then deembedded from the transistor measurements to allow access to the extrinsic shell. The extrinsic network is then identified using Cold-FET techniques [2]-[4] using a new technique including optimization over a broad frequency range.

\section{Manifold Modeling \& De-Embedding}

As a starting point consider the layout shown in Fig. 2 . This diagram shows the metallization in a typical test structure used for transistor model development. In practice several such structures will be used to generate the model. Layout parameters include number of gate cells, cell pitch, and transistor cell width. What is needed is a practical approach to segment the problem such that the result provides the requisite accuracy. 


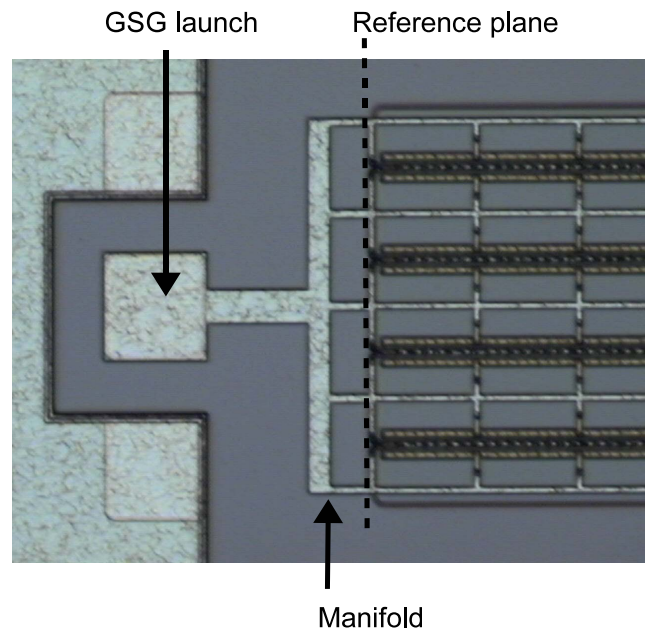

Fig. 2. Discrete LDMOS transistor used for device modeling; this structure is GSG probe-able, and the reference planes for the manifold are shown.

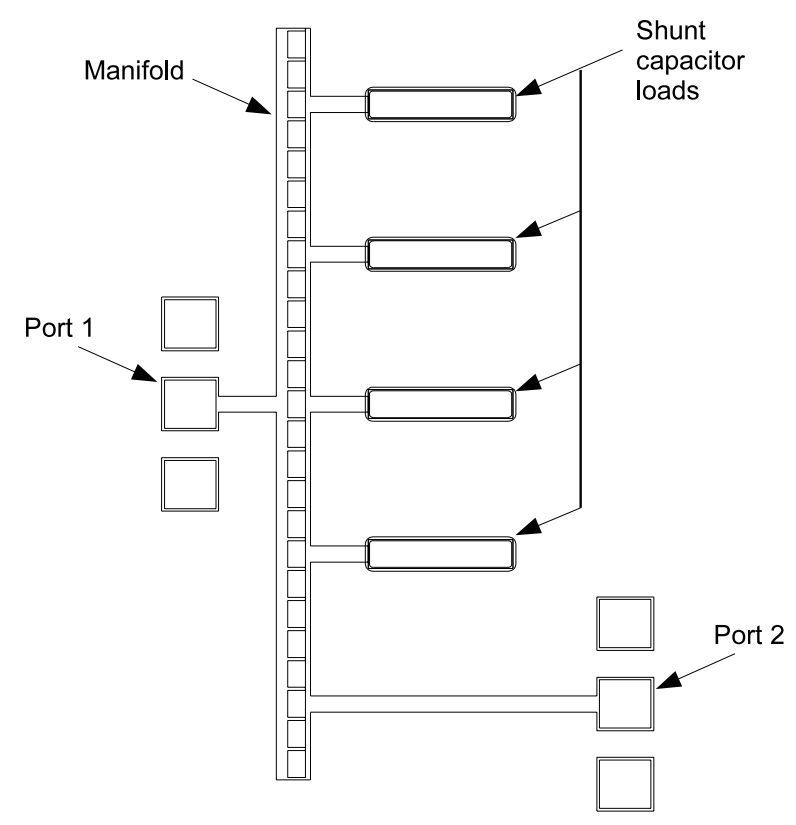

Fig. 3. Manifold test structure for electromagnetic simulation showing shunt capacitor loads.

The problem is segmented into three distinct areas of analysis, viz the ground-signal-ground (GSG) launches, the manifolds, and the extrinsic elements within the core of the transistor. The goal is to remove the effects of the launch and manifolds, leaving only the response of the transistor including extrinsic elements. The effect of the launch is removed by construction of error boxes. This is done using measured data of open and thru structures. We note that LDMOS is typically built on thin, heavily doped silicon substrates. 'Thin' means that the thickness of the silicon bulk is comparable, or less than, the skin depth at the frequency of operation.

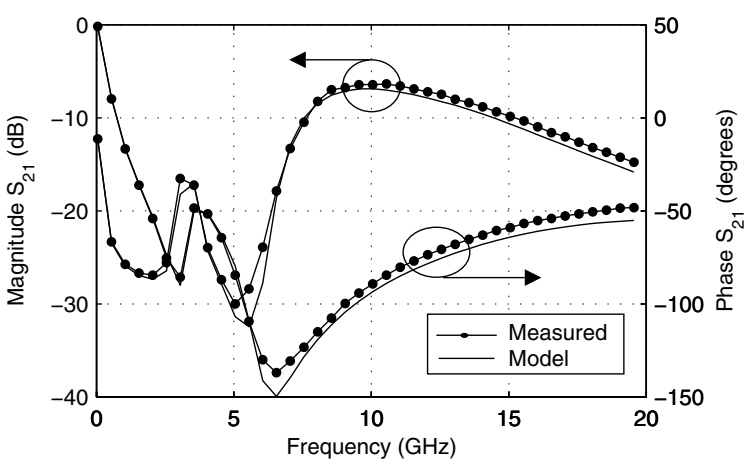

Fig. 4. Measured versus EM-modeled transmission characteristics for the structure shown in Fig. 3, indicating excellent broadband agreement.

Consequently the series impedance of the GSG has appreciable resistance due to induced eddy currents in the silicon. The shunt resistance of the ground contacts to the backside metallization is another source of added resistance.

The behavior of the manifolds in turn is obtained through electromagnetic simulation. Using Sonnet's $\mathrm{em}^{\mathrm{TM}}$, a commercially available planar electromagnetic simulator based on the method-of-moments (MoM), a substrate definition is determined by careful analysis of measured S-parameters for transmission lines of various lengths and widths. This is done by converting the $\mathrm{S}$-parameters to the telegrapher's parameters for each line and using that information to determine the properties of each layer, and the metal model which best describes the data. Once determined, the substrate definition is used to simulate S-parameters for each of the manifold structures. For the results presented here, the same wafer used for transistor modeling included structures for determination of the simulation substrate definition.

When simulating the manifold, it is necessary to choose appropriate reference planes for the generation of S-parameters. The measurement and extraction of each GSG launch error box puts one error box port exactly at the end of the thru. The other reference plane is was set at the edge of the transistor active area, as indicated in Fig. 2.

To validate the procedure, measured S-parameters of a loaded manifold were compared to a circuit simulator result for simulated manifolds plus measured loads. Large manifold 2port GSG structures were built, both with capacitive loads and unloaded. A typical structure is illustrated in Fig. 3. The loads were shunt capacitors placed periodically along the manifold width, with capacitance matching the effective capacitance of a typical LDMOS device under bias. S-parameters of a single such capacitor were also measured directly. Electromagnetic simulations of the manifolds were combined with the measured load S-parameters in a circuit simulator.

The two-port measurements of the loaded manifold structure, illustrated in Fig. 3, are in good agreement with simulations. The transmission through the loaded manifold structure is plotted against measured results in Fig. 4. Although not plotted the remaining S-parameters are in similar agreement. 


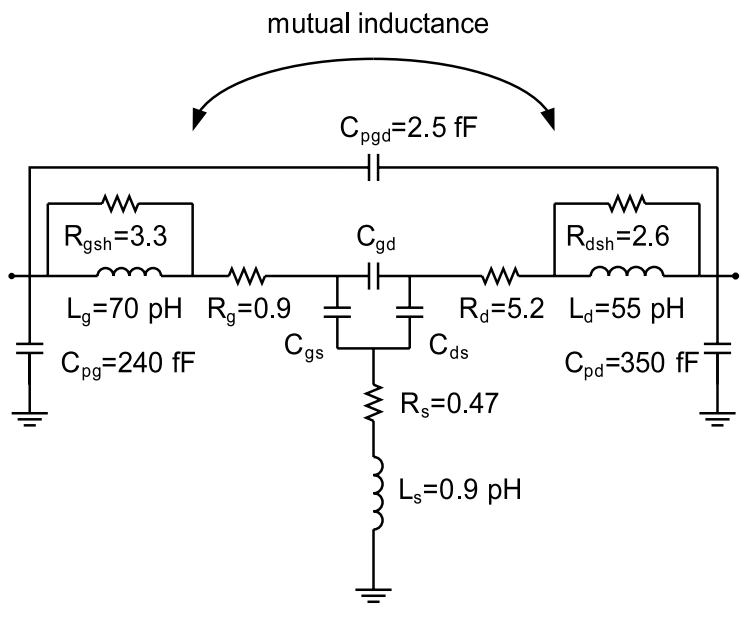

Fig. 5. The new extrinsic network with the Cold-FET intrinsic circuit also shown.

Now that we have demonstrated an accurate methodology to move the reference plane, by de-embedding the manifolds, we direct our attention towards extracting the extrinsic parameters.

\section{Extrinsic Network And PARAmeter Extraction}

The traditional extrinsic network for a FET comprises a series resistor-inductor network connected to each gate and drain, and source nodes of the intrinsic model - the Z-shell, followed by shunt capacitors from gate and drain to ground, and between gate and drain - the $Y$-shell [2]. This arrangement works well for small FETs in III-V technology.

For LDMOS transistors we have augmented this simple network with a resistor in parallel with the series gate $\&$ drain inductance, to account for the frequency dependent behavior of the silicon substrate material in an empirical manner. Adding these shunt resistors enables an improved broadband fit of the return losses. Further, we have observed coupling effects between the gate and drain metallizations in these power transistors. The unit gate widths in these power transistors are quite significant, over $500 \mu \mathrm{m}$, and there is measurable mutual inductance between these long metal traces.

The Cold-FET method is the basis for our extrinsic parameter extraction. Broadband S-parameters were measured under Cold-FET bias conditions of zero volts on gate and drain terminals of the transistor. The manifolds were then deembedded from these data by using their S-parameter blocks determined as described in Section II. The Cold-FET circuit for the intrinsic transistor is assumed to be a purely capacitive network. The extrinsic network and Cold-FET circuit are illustrated in schematic form in Fig. 5.

To extract the extrinsic network parameter values, we used the following sequence of actions: first, de-embedding the extrinsic network, then calculating the intrinsic (Cold-FET) parameters, and finally re-embedding this intrinsic model with the extrinsic network. These model S-, Y-, and Z-parameters were compared to the measured values (after de-embedding of the manifolds). The extrinsic network parameters were

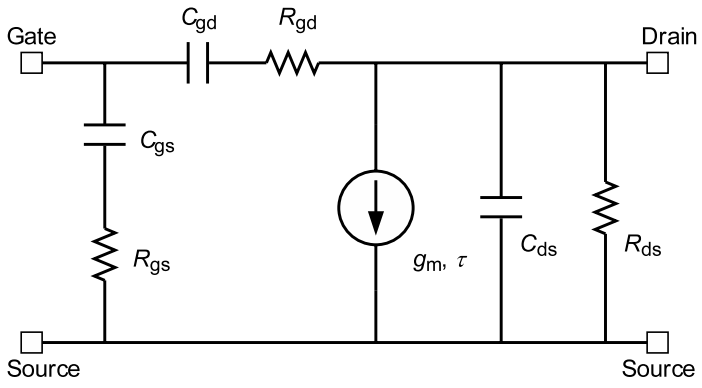

Fig. 6. The intrinsic small-signal equivalent circuit model used in this work.

adjusted until the measured and model data were suitably close, over the broadband frequency range. This procedure was performed in Agilent-EEsof $\mathrm{ADS}^{\mathrm{TM}}$, using the Sequencer component and the Tuning simulator. As a starting point, either nominal or zero values for the extrinsic network components can be used with equal success, indicating the robustness of the technique.

In particular, the resistive elements of the extrinsic network were adjusted until the low-frequency values of the real part of the model Z-parameters agreed with the measured (deembedded) Z-parameters. The gate and drain shunt capacitances were adjusted to match the low-frequency values of the imaginary parts of the Y-parameters. The gate inductance and shunt resistance were adjusted to match the broadband $S_{11}$ and real part of $Z_{11}$; and the drain inductance and shunt resistance were adjusted to match the broadband $S_{22}$ and real part of $Z_{22}$. The source and mutual inductance were adjusted to fit the broadband $S_{12}$ and real part of $S_{12}$.

Once good broadband agreement between the Cold-FET model and measured S-parameters was obtained, the prototype extrinsic network was saved. The procedure was then repeated using broadband S-parameter data measured under active transistor bias conditions. Typically we use three Class$\mathrm{AB}$ bias conditions for power transistor characterization. The objective of this second exercise is to modify the extrinsic parameter values as necessary to maintain good broadband agreement between measured and modeled S-parameter, and also to ensure that the values of the small-signal intrinsic parameters are physically realistic. We use the simple smallsignal model shown in Fig. 6, and use the equations for parameter extraction provided in Ref. [2], as these are also used in the large-signal model extraction. In particular, real and positive values for the extrinsic parameters are required for the small-signal model to be meaningful, and the largesignal model to be extractable.

Generally, we have found that the modifications to the extrinsic parameter values determined from the Cold-FET data are quite small for the active bias data to be well-matched and the model values to be physically realistic. The broadband $\mathrm{S}$ parameters for the small-signal model and extrinsic network are compared to the measured and de-embedded S-parameters in Fig. 7, showing excellent agreement. 

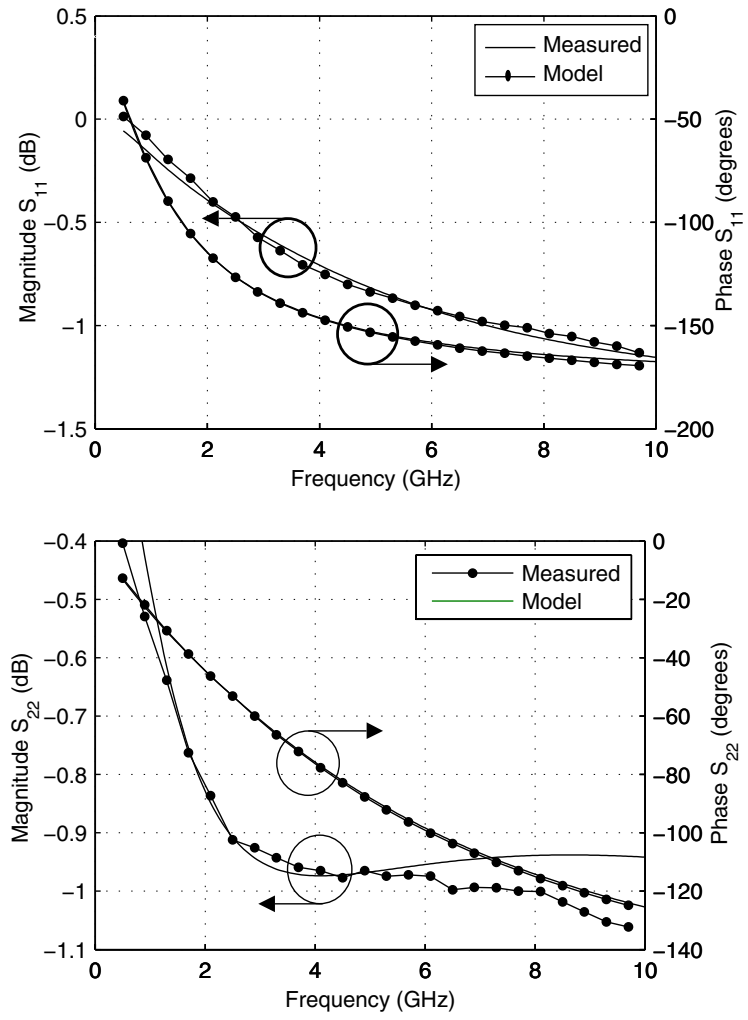

Fig. 7. Comparison between measured and modeled manifold-de-embedded S-parameters.

\section{SCALING Considerations}

As noted earlier, the scaling behavior of the complete transistor model is heavily dependent on the scaling of the extrinsic network. As the extrinsic network captures the electrical behavior of the transistor layout, the scaling performance of the extrinsic network parameters is a good test of how well this electrical behavior is modeled. For RF power transistors, the unit gate width is generally maintained constant, and scaling of the device is simply by the number of gate fingers.

The extrinsic network parameters were found to scale with total gate width in either a linear or inverse linear manner. Such simple and direct scaling indicates that the extrinsic network captures the electrical characteristics of the device layout very successfully.

\section{VERIFICATION}

The accurate matching of the small-signal model parameters with the measured and de-embedded data over the broad frequency band gives us confidence that the extrinsic network has been identified accurately. However, RF power transistors are usually employed under high power and large-signal conditions of operation. Therefore, it would seem prudent that any verification of the extrinsic network model should include a large-signal measurement and simulation. Typically, RF power transistors are characterized using load-pull techniques at high output powers.

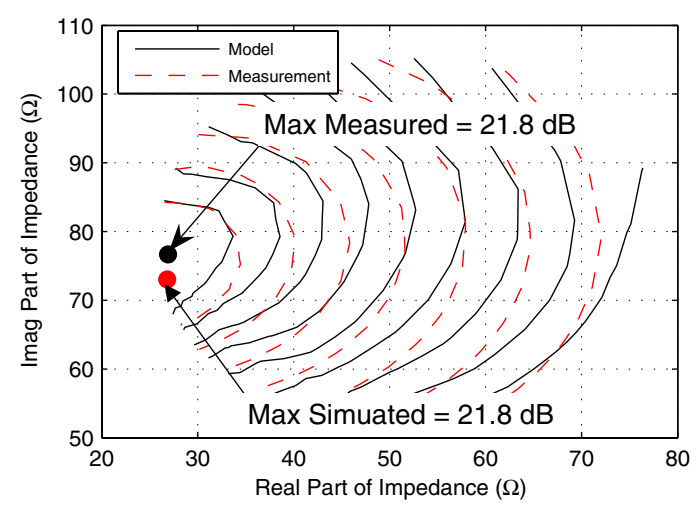

Fig. 8. Comparison between measured and modeled transducer gain load-pull contours.

In Fig. 8 we show the measured transducer gain contours for a $4.8 \mathrm{~mm}$ gate-width LDMOS power transistor operating in Class-AB bias, taken at $2.14 \mathrm{GHz}$. In this figure we also show the predicted contours from the industry-standard Motorola Electro-Thermal (MET) model with the new extrinsic network. The model matches the contours and peak gain point with excellent accuracy over the range of output powers and load impedances.

\section{Conclusions}

We have described a new extrinsic network for RF power LDMOS transistors, and outlined a procedure for estimation of the network parameter values. The new network is designed to accommodate features attributable to the lossy silicon substrate, and to the layout of the power transistor. The parameter extraction procedure takes advantage of sequencer and tuning capabilities of the circuit simulator to identify the network parameter values over a broad frequency range. The new network was observed to scale with gate width effectively and simply, and large-signal verification shows excellent performance when coupled the industry standard MET model for the intrinsic device.

\section{ACKNOWLEDGMENTS}

Thanks are due to the management staff of RF Division, Freescale, in particular Jaime Plá, for encouraging and supporting the development of this new model

\section{REFERENCES}

[1] P. H. Aaen, J. A. Plá, and J. Wood, Modeling and Characterization of RF and Microwave Power FETs. Cambridge, UK: Cambridge University Press, 2007.

[2] G. Dambrine, A. Cappy, F. Heliodore, and E. Playez, "A new method for determining the FET small-signal equivalent circuit," IEEE Trans. Microwave Theory Tech., vol. 36, no. 7, pp. 1151-59, July 1988.

[3] D. Lovelace, J. Costa, and N. Camilleri, "Extracting small-signal model parameters of silicon MOSFET transistors," in IEEE MTT-S Int. Microwave Symp. Dig., San Diego, CA, May 1994, pp. 865-868.

[4] J. Wood and D. E. Root, "Bias-dependent linear scalable millimeter-wave FET model," IEEE Trans. Microwave Theory Tech., vol. 48, no. 12, pp. 2352-2360, Dec. 2000. 